\title{
Idealizations in empirical modeling
}

\author{
Julie Jebeile \\ IRFU/Service d'Astrophysique, CEA Saclay, 91191 Gif-sur-Yvette, France \\ julie.jebeile@gmail.com
}

\begin{abstract}
In empirical modeling, mathematics has an important utility in transforming descriptive representations of target system(s) into calculation devices, thus creating useful scientific models. The transformation may be considered as the action of tools. In this paper, I assume that model idealizations could be such tools. I then examine whether these idealizations have characteristic properties of tools, i.e., whether they are being adapted to the objects to which they are applied, and whether they are to some extent generic.
\end{abstract}

\section{Introduction}

In empirical modeling, mathematics has an important utility in transforming descriptive representations of target system(s) into calculation devices, thus creating useful scientific models. Descriptive representations in this context are pieces of knowledge about the properties and the behavior of target system(s) which are not yet expressed in mathematical terms. They are one form of models that, when mathematized, become inferentially useful. Mathematics thus allows models to fulfill their inferential role which can be to predict, explain or design experiments. In other words, if they are not mathematized, models would be partially descriptive representations of little impact and use.

Because mathematics here transforms descriptive representations into calculation devices, the transformation may be considered as the action of a tool or, more exactly, of several tools. In this paper, I assume that the idealizations involved in the transformation going from descriptive representations to a useful model could be such tools. I then examine whether these idealizations have the usual expected properties of tools, i.e., whether they are being adapted to the objects to which they are applied, and whether they are to some extent generic.

Ordinary tools - such as pliers, screwdrivers and wrenches - transform the objects on which they are used for a given purpose. They do so by virtue of having physical properties which are adapted to the objects ${ }^{1}$. That said, tools cannot be id-

\footnotetext{
${ }^{1}$ Mutual adaptedness seems to be a specific property of some ordinary tools. Here, not only the tool is adapted to the object on which it is used, but also the object is adapted to the tool. This is a case of screwdrivers and screws, Allen keys and bolts, or hammers and nails. Some ordinary
} 
iosyncratic (or they would lose their utility) and must rather apply to a certain range of objects. For example, an Allen key is a tool of hexagonal cross-section that is used to drive a range of bolts and screws as soon as they have a hexagonal socket in the screw head. What about idealizations in empirical modeling?

One might hope that, by contrast, mathematics applies identically to descriptive representations whatever they may be. In this way, it would be a very efficient tool in the modeling field because it would be universal. I will put forward the claim that this cannot be true and assess to which extent the transformation of models into mathematical terms is actually adapted to the specific empirical nature of the target system(s).

In this paper, I will first describe how models are built, focusing on their transformation into mathematical terms. This transformation has two phases: the first is about expressing the initially representational content of the model in mathematical equations. The second is about making these equations tractable. As we will see, idealizations are involved in both phases of transformation.

I will then argue that, for the model to be useful, its transformation into mathematical terms has to be adapted to the target system(s). Thus, the idealizations involved in the process must be constrained by the specificity of the target(s). Not only are these idealizations designed for models to be inferential, but they must also be chosen in such a way that models preserve at least a minimum amount of relevant accurate information about the systems under study. For my argument to be general enough, I will consider transformations that result in both analytically solvable and numerically solvable models.

That said, I will further argue that adaptedness here does not mean restriction to the sole systems under study. I will show that the idealizations involved in a transformation may well be suited for modeling other empirical systems as well. In that sense they function as mathematical tools that have a certain scope of application, as one would expect from tools in the ordinary sense of the word.

\section{Model building}

In this section, I will describe the process of building models and will place special emphasis on the transformation into mathematical terms. My aim is to show that idealizations play a central role in this transformation.

I shall specify that my way of describing model building differs dramatically from the mapping accounts although they are important contributions in the discussions about the role of mathematics in empirical modeling (e.g., Batterman 2008, Bueno and Colyvan 2011, Pincock 2007). As the proponents of these accounts identify something called the mathematical structure of models, they treat as one the formal feature and the representational content of models. It may be a

tools do not share this property, however (e.g., rakes, scissors and shovels). Because this property is specific, it will not be considered in this paper. 
relevant philosophical approach to study in some way the mathematical role in scientific models. Here, however, I want to emphasize and to characterize the action of mathematics as a tool on a descriptive representation to make it a calculation device. Thus, a diachronic presentation of model building is preferred instead.

In this presentation, I offer a conceptual account of the stages in empirical modeling. It is inspired by Cartwright' account of theory entry proceeding (1983, Essay 7) but also differs from her account in that hers is more descriptive (mine is more idealized). According to Cartwright, scientists start with an unprepared description which contains everything they know about the system under study, including information they think relevant in whatever available form; information can be theoretical components, observations or measurements. From this a prepared description is established in preparation for mathematically formalizing the physical phenomenon under study. It contains theoretical principles applied to idealized cases, as well as boundary conditions and ad hoc terms used to match the empirical data.

The account of empirical modeling, as proposed here (see figure 1), is a revised and idealized version of Cartwright's conception in that it re-scales the transformation into mathematical terms within empirical modeling. In this account, I deliberately magnify the mathematical process so that it becomes clear how mathematics actually transforms initially descriptive representations into models.

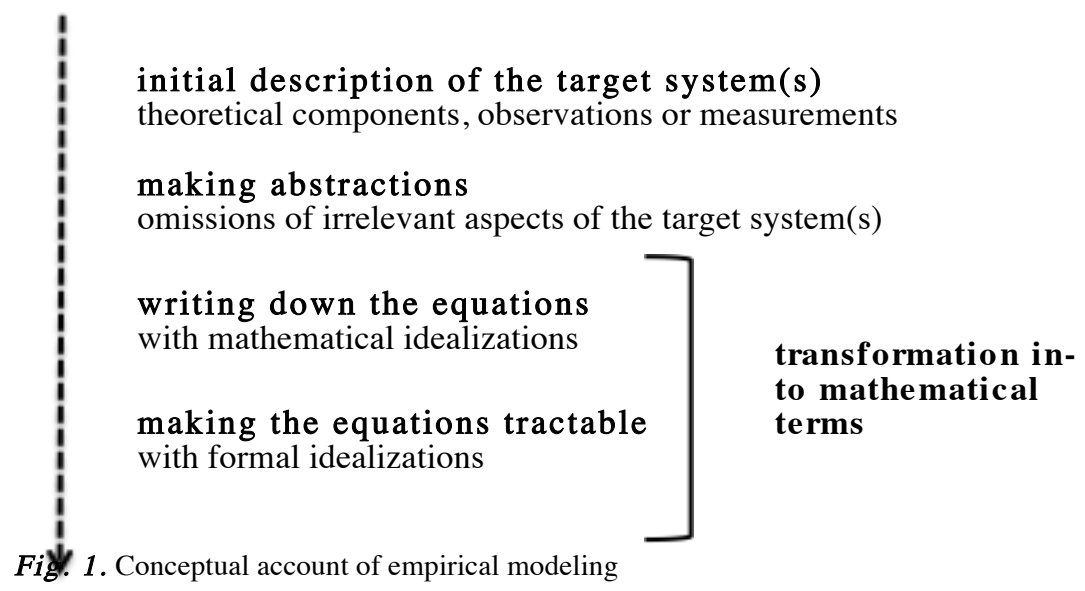

In my account, I suggest that, at the very beginning, model building consists of an initial description of the target system(s) involved in the phenomenon under study. The situation is likely to be the same as what Cartwright describes as the phase of the unprepared description, except that, here, the description is supposed to still not have a mathematical form.

Then, scientists have to make a choice among all the information within the initial description, and select the relevant aspects about the phenomenon under study to be included in the model that is to be built. In other words, they have to make 
some abstractions ${ }^{2}$. Without this selection, modeling would not be possible. For Godfrey-Smith (2009), abstracting is required whatever the nature of the description may be (scientific or literary, for example) because there is no useful description which would exhaustively contain all the aspects of a system. For instance, there is no point in specifying the Moon phase for describing the motion of a body, or, of including the presence of oxygen or the average temperature for describing the trajectory of planets ${ }^{3}$.

The description, once cleared of the less relevant details, contains all the relevant representational aspects but does not yet have the desired inferential power (of predicting, explaining or designing experiments) ${ }^{4}$. For the model to be a calculation device, this description must be transformed into a useable set of mathematical equations. This corresponds to what I have earlier called the transformation of models into mathematical terms.

The transformation (which could be also called mathematization) consists in two phases: the first is about expressing the initially representational content of the model in a first set of mathematical equations, and the second is about making this set of equations tractable. Both phases are necessary for the model to become a calculation device. The first phase aims at creating a preliminary version of the calculation device, and the second aims at making this device useable for inferential tasks. That said, building a mathematical model often starts from another mathematical model. This situation can be expressed as feedback loops in my schema of modeling phases.

The transformation involves, in its two phases, idealizations, which are simplifying assumptions expressed mathematically in the equations ${ }^{5}$ (see e.g., Cartwright 1983; McMullin 1985; Hacking 1983; Laymon 1980, 1985, 1989a, 1989b, 1995 for discussions on idealizations):

In the first phase, the expression of equations consists in translating the initially representational content into a mathematical language, i.e., in terms of mathematical symbols (e.g., numbers, variables, constants, vectors, matrix), operations (e.g., addition, integration, derivation) and functions (e.g., cos, sin, log). This translation

\footnotetext{
${ }^{2}$ Abstractions differ from idealizations in that they are omissions of some aspects in the target system which are not relevant for the problem being studied (e.g., to neglect gravitational force in subatomic phenomena), whereas idealizations are distortions (Jones 2005; Godfrey-Smith 2009). Idealizations can be omissions but, in this case, these omissions distort the representation in that they are omissions of relevant aspects.

${ }^{3}$ Abstracting can sometimes later be part of mathematization. For instance, difficulties in formulating equations might occur and lead to a different abstraction. This is, however, an additional aspect that I do not treat in this paper.

${ }^{4}$ What is relevant might actually depend on the final success in constructing a useful model, and therefore be identified as such at later stages of modeling. In such cases, there might be some back and forth in the process of model building.

${ }^{5}$ I shall stress that this way of defining idealization differs from the view on which model as a whole is an idealization. It is considered here that idealizations are only parts of a model. Unlike models, idealizations have no inferential power on their own. For instance, the Ising model will not be considered as an idealization but as being composed of idealizations. A mass point is an idealization, but is not a model in that, alone, it has no inferential power.
} 
is required because mathematical language enables to perform afterwards the expected inferential tasks. Mathematical language is generic in that the symbols, the operations and the functions may well be used to describe any empirical system. It therefore constitutes the formal part of the model that is clearly not specific to the system(s) under study.

That said, the linguistic translation of the initially representational content requires to make mathematical idealizations which fit this content to the constraining adopted mathematical language. Mathematical idealizations can be about taking some of the spatio-temporal properties out of the target system(s), thus abstracting away the "imperfections" of matter from the target system(s), for the final description of these systems to match ideal geometrical forms. For example, a wooden wheel or a balloon may be represented as a perfect circle.

The equations obtained in the first phase may be intractable - either analytically or numerically. In this case, one must proceed, in the second phase, to formal idealizations ${ }^{6}$. This is done either by considering the physical problem under study, or by considering the mathematical form of the equations.

In the former case, one tries to simplify the physical problem by omitting or by distorting some aspects of the system(s) in order to get results from calculation: "Complicated features of the real object(s) are deliberately simplified in order to make theoretical laws easier to infer, in order to get the process of explanation under way" (McMullin 1985, p. 258). Examples of formal idealizations are results from considering a body as being a mass point, an infinite surface or a frictionless surface, from replacing non-linear interactions by linear interactions, or from assuming an oscillation as being small. In some cases, considering the physical problem and trying to simplify its description are, however, not enough.

Because the equations remain intractable or complicated to solve, additional formal idealizations - which are sometimes called "approximations" in this context (Ramsey 1990, 1992; Laymon 1989a, 1989b) - are conceived based on the mathematical form of the equations. Here, formal idealizations do not always correspond with familiar idealized physical situations. Let us consider the example of a body of unit mass falling in a weakly resisting medium ${ }^{7}$. It is described by $\mathrm{d} v / \mathrm{d} t$ $=\mathrm{g}-\mathrm{k} v$, with $\mathrm{g}$ the acceleration due to gravity and $\mathrm{k}$ a friction coefficient. Let us assume that the speed is zero when the body starts to fall $(t=0)$. Then $v(t)=$ $(\mathrm{g} / \mathrm{k})(1-\exp (-\mathrm{k} t))=\mathrm{g} t-\mathrm{gk} t / 2+\mathrm{gk}^{2} t / 6-\ldots$. When the friction resistance is insignificant (i.e., $\mathrm{k}$ is small), the terms with $\mathrm{k}$ can be removed and the speed is approximately described by the first term in the power series $v(t)=\mathrm{g} t$. This formal idealization corresponds to a conceptually well-identified distortion-i.e., the absence of air - that is associated with a familiar idealized physical situation-i.e., a free fall in vacuum. That said, a formal idealization may consist in removing the terms after the second or the third order (or any superior order) of the power series. Here, the idealization would hardly correspond with a familiar idealized physical situation. In our language and division of the world into concepts, we

${ }^{6}$ I borrow the distinction mathematical vs. formal idealizations from McMullin (1985).

${ }^{7}$ I take this example from Norton (2012) for a different purpose though. 
lack an expression to identify an aerial space which exerts on a body a resistance such that its speed equals exactly $\mathrm{g} t-\mathrm{gk} t / 2+\mathrm{gk}^{2} t / 6$, for example.

In a nutshell, mathematical idealizations make it possible to express the model in terms of mathematical equations. Formal idealizations are required to make these equations tractable and thereby useable. Without mathematical and formal idealizations, the model would not be a calculation device but merely a partial description of the world.

I have shown how important idealizations are in the transformation of models into mathematical terms. It follows that the transformation is adapted to the empirical nature of the systems under study if the choice of the involved idealizations depends on the specificity of the systems. In the next section, I will contend that these idealizations should actually be constrained by the nature of the systems so that the transformation should be adapted to it.

\section{Adaptedness of idealizations}

I will now argue that idealizations are tool-related in that they make the model useable. I will further argue that they are adapted to the kind of phenomena to which they are applied if the model is expected to be not merely useable, but useful.

Idealizations are tool-related. Following Carrier and Lenhard, this means that "they result from the properties of the tool and make sure that the tool can be used in the first place" (cf. the general introduction). Let me briefly clarify in what sense idealizations are tool-related. In the transformation of models into mathematical terms, idealizations are used to shape initially representational components into a mathematical form, so that models become tractable and therefore usable. In constraining the representation by a mathematical language, idealizations give models a new property, i.e., inferential power. In other words, idealizations make it possible to create a device from which calculations can be done.

I suggest that their capacity to give models inferential power results from the fact that they essentialize the features of the target(s) that they denote. They essentialize in the sense that they reduce the features to something formally and representationally essential. It is formally essential in that it is a mathematical object making tractable the equation that contains it. It is representationally essential in that it represents a feature of the target that is relevant for building the representation one needs.

One might think that, since idealizations are tool-related, they must meet mere mathematical constraints but no representational constraint. In the first phase, mathematical constraints are due to the mathematical language that the model must fit. In the second phase, mathematical constraints are due to the available mathematical means of solving equations (i.e., whether we know how to solve them). This would be true if the model is expected to be useable. A model is useable in that it is mathematically tractable and can be used to make calculations and 
to provide results. That said, in empirical science, a model is expected to be more than useable; it should be useful. A useful model has to be useable and has to provide reliable results for answering the questions the scientist is asking. My aim is to show that, for a model to be useful, the choice of the idealizations involved in the transformation must depend on the nature of the target systems.

Idealizations transform the initially representational content from the unprepared description into a calculation device by distorting this representational content. Thus, models become calculation devices to the detriment of their representational function. That is why a lot of philosophical attention has been paid on whether, once idealized, models can teach us something about the world (e.g., McMullin 1985; Laymon 1989a, 1989b; Bokulich 2008, 2009; Strevens 2007; Weisberg 2007). Thus, idealizations are internally stressful concepts. Employing idealizations involves a trade-off between making a model useable and making a model useful. Therefore, the scientist has to find a compromise when choosing the idealizations to be included in the model.

For a model to be useful, I suggest that idealizations must be selected in a way that makes the model sufficiently accurate for the purpose at hand. I thus follow van Fraassen when he writes that "A representation is made with a purpose or goal in mind, governed by criteria of adequacy pertaining to that goal, which guide its means, medium, and selectivity" (2008, p. 7). He further claims that a "representation useful for particular purposes will involve selective distortion, and representation is closely involved with useful misrepresentation. Even when likeness is crucial to the purpose, we must look for likeness only in respects that serve the representation's purpose - and only to the extent that they do so" (2008, p. 87). Hence, idealizations that are the distortions in a model are allowed to the extent that they are selected adequately for the purpose at hand.

I will show that the choice of idealizations relates to issues of representation, and choosing an adequate idealization hinges on considerations about the specific empirical nature of the target system(s). As I will elaborate with the example of Prandtl's model, the choice of adequate idealizations is constrained by the specificity of the targets. Idealizations must not make the model deviate too much representationally from the actual description. Thus, I will claim that not only are idealizations in the transformation tool-related but they are also object-related. Object-related idealizations "create a simpler version of the relevant objects and their relationships so that mathematical models of them become more tractable" (cf. the general introduction) and, I shall add, so that mathematical models become of real utility in empirical science.

Let me illustrate this with the story of Prandtl's model. The study of a flow past an obstacle has long been an important industrial issue, dating back to the rise of steamboat navigation during the Victorian times. It was imperative that British engineers know how to assess the resistance of water on a boat in order to design optimal hull shapes. However, such a study is mathematically difficult. D'Alembert (1782), as well as other physicists and engineers studying fluid mechanics such as Poncelet, Saint-Venant, Boussinesq, Ranquine or Froude, suggested, tested, and 
tried to improve models of fluid resistance. But all of them faced the same thorny problem: one of the required idealizations leads to unacceptable aberrations.

Two important formal idealizations were introduced in the models. The first was an incompressible flow condition, which leads to the assumption that the influences of pressure and temperature on mass density are negligible. This idealization was unproblematic. The second idealization neglected all effects of viscosity (i.e., internal friction in the fluid). This idealization seems prima facie justified for applications involving fluids like air and water, which have a low viscosity. It results in equations with explicit solutions, which are known as the Euler equations. Thus, the idealization helps to provide a useable model. Nevertheless, the Euler equations lead to absurd results, at least if interpreted physically. One such absurdity, discussed by d'Alembert, is the cancellation of the drag of a moving body. In other words, there would be no force exerted by the fluid on the body, which contradicts our most familiar observations: air slows down a balloon that was initially thrown horizontally, for example. The Euler equations raise other difficulties since they cannot provide explanations of phenomena such as pressure loss in a pipe or separating flow past an obstacle. These phenomena are the result, directly or indirectly, of fluid viscosity. The no-viscosity assumption is therefore too strong, even when modeling low viscosity fluids. It is not a harmless idealization in the sense of Elgin and Sober (2002); it jeopardizes the representational adequacy of a model that contains it.

If the idealizations in the transformation were only constrained by mathematics, such an issue would not appear. This example illustrates that, for a model to be useful, idealizations need to be sensitive to the nature of the target system(s). Thus, other idealizations are required which take into account viscosity in low viscosity flows. What are they?

With the objective of solving d'Alembert's paradox in mind, Navier and Stokes both contributed in establishing the fundamental equations of Newtonian fluid mechanics in a continuous medium in which they both introduced terms of fluid viscosity. In 1845 the final version of the Navier-Stokes equations was established. These equations derive from balances of mass, momentum, total energy and entropy applied on a fixed or mobile volume of fluid. Unfortunately, they form a complex system of equations with non-linear partial derivatives whose analytical resolution is still today a real challenge for mathematicians. Their resolution is one of the seven Millennium Problems raised by the Clay Mathematics Institute.

In order to get a useful model that is both inferential and representationally good enough, idealizations must be found which are more subtle than the noviscosity assumption. Prandtl's model illustrates this achievement. Before Prandtl's model, successfully applying the Navier-Stokes equations was practical-

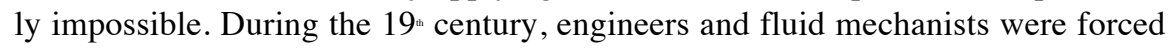
to establish phenomenological laws in order to solve their problems (Darrigol 2005). More than 150 years after the first work of d'Alembert (1782) discussed the forces exerted by a fluid on a solid body, Prandtl made a significant contribution in the use of these equations. His model is explicitly presented as a means of 
applying the Navier-Stokes equations to the concrete problems of a flow past a solid (Heidelberger 2004). Ludwig Prandtl himself introduced the model in 1904 in these terms at the third international congress of mathematicians at Heidelberg (Darrigol 2005, p. 283).

In his model, Prandtl assumed the influence of forces induced by low viscosity as confined in the wake behind the obstacle as well as inside a layer, i.e., the boundary layer. Low viscosity fluid around an obstacle is conceived of as the interaction between two components. The first component is the boundary layer which is around the obstacle and whose viscosity is not zero and whose velocity profile evolves linearly. The velocity profile starts from zero at the interface fluid/obstacle - this is called the no slip condition-and reaches a maximal value equal to the viscosity in the second component. The second component is the wake. Within the wake, the viscosity is zero and the mean velocity remains constant. For this component alone, the Euler equations apply. With such an idealization, it has been possible to describe the trajectories of bodies in a flow-e.g., plane flight in the atmospheric air-or the fluid flow past an obstacle-e.g., flow of a river against a boat - , and thus to study hydrodynamic behaviors such as the onset of vortices.

The idealization conceived by Prandtl is more subtle than the simple omission of the terms of viscosity in the Euler equations. Idealizing for building Prandtl's model is not about automatically omitting or modifying certain terms in the Navier-Stokes equations that do not meet mathematical expectations. Because, even if the boundary layer is thin in ordinary fluids such as water and air, it has significant effect on flow in virtue of the high gradient of velocity that it implies. In this case, the right idealization is constrained by the specificity of the system being studied. Here the specificity is that the viscosity, albeit low, plays a crucial role to describe drag, pressure loss or flow separation. Understanding the relevance of this physical aspect is more important than merely considering its low numerical value, for instance. In other words, for a model to be useful and not just useable, it seems that idealizations cannot be made without considering the physical problem.

Another aspect of the story reinforces the idea that idealizing properly requires the consideration of the specificity of the physical problem. The concept of boundary layer was obtained by Prandtl through observations, i.e., visualizations of experiments that he conducted in a water tunnel. Morrison claims that since the boundary layer concept is the product of direct observations, it is what she calls a phenomenological abstraction. She writes indeed that "The model is phenomenological not because there is no theory from which to draw but because it is motivated solely by the phenomenology of the physics; in fact, once the model is developed theory does play an important role in its application" (Morrison 1999, p. 54). It seems that Prandtl even developed a certain intuition through the visualization of the phenomena before even setting the equations of his model. On that subject, Prandtl says: "Herr Heisenberg has [...] alleged that I had the ability to see without calculation what solutions the equations have. In reality I do not have this ability, but I strive to form the most penetrating intuition [Anschauung] I can of the things that make the basis of the problem, and I try to understand the process- 
es. The equations come later, when I think I have understood the matter" (quotation taken from Darrigol, 2005, p. 287).

The difficulty scientists may have in finding appropriate idealizations has been overlooked by the philosophical discussions about idealizations. It is rather often assumed that scientists know well in advance the empirical consequences of the idealizations contained in a model (exceptions are notably Laymon and Ramsey). A famous example is a freely falling object. It is often suggested that scientists only use the assumption of zero air resistance for predicting the velocities of heavy bodies because they know that the assumption becomes inadequate when studying low-mass objects for which air resistance cannot be neglected. (They also know that, in that case, they need to provide an approximate phenomenological term for the air resistance force.) It may be sometimes true that scientists know the scope of idealizations especially when they use them for a long time. Prandtl's model, however, exemplifies a case where it is not true. The model also shows that there is no unique recipe that may help one to automatically make the following inference: "the fluid viscosity has a low value" then "the term of viscosity can be omitted in the equations." Such a point is even made in textbooks on fluid mechanics: "Approximation is an art, and famous names are usually associated with successful approximations: Prandtl wing theory, Kármán-Tsien method for airfoils in subsonic flow, Prandtl-Glauert approximation for subsonic flow, Janzen-Rayleigh expansion for subsonic flow, Stokes and Oseen approximations for visas flow, Prandtl boundary-layer theory, Kármán-Opohlhausen boundary-layer approximation, Newton-Busemann theory of hypersonic flow" (Van Dyke 1975, p. 2).

In a nutshell, not only are idealizations designed for models to be inferential, but they also must be chosen in such a way that models preserve at least a minimum amount of relevant accurate information about the systems under study. Hence, this indicates that the idealizations in the transformation have to be both tool-related and object-related. Thus, the transformation is adapted to the nature of the target(s).

In the next section, I will generalize this claim in studying different transformations of the representation of a same phenomenon. I shall emphasize that distinct transformations are related to distinct possible tasks.

\section{Plurality of transformations}

The computational turn, which is the historical conjunction between the development of numerical analysis and the advent of computers, offers new means of writing and solving model equations, i.e., new transformations. It is possible to use numerical methods for expressing and solving equations. In this section, I will consider the case where different transformations are used to build a model of a same target system. In this descriptive part, I will emphasize, through the study of a flow past a cylinder, that each transformation involves its own idealizations, thus being adapted in a unique way to the target system. It will follow that, since ideal- 
izations involved in a transformation are both tool-related and object-related, each transformation transforms the initially descriptive representation into a model that can be more or less adequate for the purpose at hand.

I will further investigate the example of low viscosity and incompressible flow past a cylinder. It can be studied under analytic method-e.g., Prandtl's modelor numerical methods applied on computer-i.e., discretization-based method, computational method of molecular dynamics, Monte Carlo method or cellular automata. All the models that are created based on these methods have in common that they express in their own way the fundamental principles of fluid mechanics, i.e., conservations of momentum, mass and energy (except nevertheless the Monte Carlo method which violates conservation of momentum as we will see). In these models, the problem is considered as two-dimensional. A first difference, however, is that some are based on a macroscopic description of the fluid while others are based on a microscopic description. The fluid is therefore idealized as a continuous medium for some models and as a set of discrete entities for the others. Let me further present the five transformations in the following.

\section{Transformation 1: Prandt1's mode1}

When they describe the fluid macroscopically, hydrodynamics models necessarily contain approximate versions of the Navier-Stokes equations. Since these equations describe the behavior of the fluid idealized as a continuum, as said before, they derive from balances of mass, momentum, total energy and entropy applied on a fixed or mobile volume of fluid. Thus, they contain the fundamental principles of fluid mechanics. Two additional idealizations are often used. First, a boundary condition near the cylinder is established, which is the no slip condition: at the interface with the obstacle, the fluid velocity is supposed to be zero. Second, the fluid is considered as incompressible.

The system of equations thus obtained-composed of constraint equation, boundary condition and incompressible flow assumption-cannot be solved as such. Indeed, the system contains non-linear terms. A first analytical approach allows one to solve it nevertheless. This is the Prandtl model. As seen earlier, in this model, the fluid is supposed to have two interacting components. The first component is the boundary layer which is around the obstacle and whose viscosity is not zero and whose velocity profile evolves linearly. Within the second component, i.e., the wake, the viscosity is zero and the viscosity is constant. 


\section{Transformation 2: discretization of the Navier-Stokes equations}

A second transformation allows one to avoid the two idealizations made by Prandtl, thus making the model more accurate. It is the numerical resolution of the Navier-Stokes equations by a discretization-based method. In this computational model, the numerical scheme is based on the integration of equations by finite element method. First, finite element method consists in discretizing the physical domain into finite elements. Then the partial differentials of the equation variables are replaced by the formal idealizations obtained with the values of the variable at the nodes of each finite element. Lastly, the obtained equations are integrated on each finite element of the meshing, and for each time step when the module of temporal dependence is required. This approach is less idealized than Prandtl's model and can therefore provide more precise results.

\section{Transformation 3: molecular dynamics model}

The macroscopic representation of fluid-on which the Navier-Stokes equations are based-is not valid if the assumption of fluid as continuum does not hold. This situation corresponds to a Knudsen number superior to $0.01^{8}$. Here a microscopic description of fluid is required. This is the case of trajectories of spatial vehicles (Meiburg 1986). Because the fluid in which these vehicles move has three different regimes: continuum fluid regime, transitional flow regime and collisionless flow regime. Yet, in the two latter regimes, the fluid cannot be considered as a continuum. Consequently, it must be represented as a discrete set of entities. In this case it is required to use the Boltzmann equation in order to describe the average behavior of fluid particles. Furthermore, it is expected that conservation of linear momentum, conservation of kinetic energy and conservation of angular momentum are satisfied. From this, two methods can be used, namely, the molecular dynamics model and Monte Carlo method.

In the molecular dynamics model it is generally assumed that there are several thousands of particles. The initial distribution of these particles is randomly determined in the space or is explicitly set. It is the same process with their initial velocities. The particles then evolve with their own velocities. They can also interact between each other following the 'potential well' model. In this model, the collision is represented as if a particle fell in a potential well, i.e., in a local minimum of potential energy. At each new time step the instant of the next collision is calculated. This is done by examining all the pairs of fluid particles. Then all the particles are moved forward at the same time in accordance with the laws of clas-

\footnotetext{
${ }^{8}$ Knudsen number is a dimensionless parameter. It indicates the flow regime depending on the fluid continuity (while Reynolds number indicates the flow regime depending on the turbulence).
} 
sical physics, and the new velocities of the particles involved in a collision are calculated (Meiburg 1986, p. 3108).

\section{Transformation 4: Monte Carlo method}

Like the molecular dynamics model, the numerical approach based on a Monte Carlo method proceeds on the calculation of the trajectories of particles. In this transformation, the fluid is represented as a lattice of cells. At the initial step, particles are randomly located on the cells or determined beforehand. Like in the molecular dynamics models, at each time step, the new positions of the particles are calculated in the space depending on their respective velocities. The Monte Carlo method differs nevertheless in the way the interactions between particles are handled. The position and the instant of a collision are here not determined by the calculation of the trajectories of the particles, but meet merely statistical considerations. In each cell, among all the particles, two particles only are selected randomly, independently of their positions. A collision between two particles is then considered: the new positions and velocities of the two particles are calculated; they are supposed to be rigid spheres rather than potential wells as they are in the case of the molecular dynamics model.

The statistical assumption that concerns the assessment of collisions makes it possible to greatly facilitate the calculations. This is the reason why, compared to the molecular dynamics model, the Monte Carlo method allows for a much higher speed of calculation running on computer. The side effect, however, is the violation of the conservation of angular momentum for the interactions between particles (see Meiburg 1986, p. 3109). In the model, recall that the calculation of the collisions is done independently of the position of the particles. One can assume that all the directions are equiprobable for the velocity of the two particles after collision. In the calculation, the direction of this velocity is therefore randomly set in selecting an azimuthal angle and a polar angle. Consequently, two components of the velocity after collision are determined by the chosen Monte Carlo method. They therefore do not remain possible variables. Moreover, the four other variables of the problem - namely, the coordinate of the velocity after collision and the three coordinates of the velocity before collision-are set when the conservations of linear momentum and kinetic energy are satisfied. Consequently, they are not available in order to meet the conservation of angular momentum. 


\section{Transformation 5: cellular automata-based mode1}

Another transformation is based on cellular automata (Rothman and Zaleski 2004; D'Humières and Lallemand 1986) ${ }^{9}$. The principle in cellular automata consists in representing the fluid as a lattice. At each node of the lattice stands a site. The state of each site received a value among a finite number of possible states. At time $t n+1$, it depends on the value of the states of the neighboring sites at time tn with which the site is connected. In the representation of the fluid, the fictive particles of the fluid possess the same mass and the same velocity. They only differ to each other in the direction of their velocity; the velocity can receive only six possible values (for a hexagonal lattice) (see Rothman and Zaleski 2004, chapter 1, p. 1-2). At each time step, the particles move from one site of the lattice to another site of the lattice following the direction of their velocity. They can collide if two particles or more arrive at the same site at the same time. Some collisions can produce the scattering of the particles. In this case, the velocity vector of the particles is modified. The total number of the particles and the sum of the velocity vectors do not change. This means that the mass and the momentum are conserved.

\section{Idealizations for the purpose at hand}

Each of the presented methods implies a specific transformation which, as I want to emphasize, involves its own idealizations:

First, the macroscopic representations-i.e., Prandtl's model and the discretized version of the Navier-Stokes equations - are in some way more idealized than the microscopic representations-i.e., the molecular dynamics model, Monte Carlo method and cellular automata-based model-in that they include the assumption of fluid as continuum.

Among the macroscopic representations, Prandtl's model is a more simplified version than the discretized version of the Navier-Stokes equations insofar as the latter does not need to assume somewhere that the viscosity is zero.

Among the microscopic representations, the Monte Carlo method includes a statistical assumption about collisions. This assumption is an idealization that the molecular dynamics model does not need to contain. Furthermore, while the molecular dynamics model and the cellular automata-based model are derived from the same fundamental physical principles of mechanics-i.e., conservations of

\footnotetext{
${ }^{9}$ The development of cellular automata is more recent than the development of differential equations since it started in the 1940s with the work of Ulam and von Neumann at Los Alamos. For a general philosophical discussion on cellular automata, see e.g., Fox Keller 2003 and Rohrlich 1990. There are also different hydrodynamics models based on cellular automata. For an exhaustive presentation of these models, see the forthcoming paper of Barberousse, Franceschelli and Imbert entitled "Cellular Automata, Modeling, and Computation" and Barberousse and Imbert (2013)."
} 
momentum, number of particles and energy - , these two models differ from each other in their degree of idealization. In the molecular dynamics model, the fluid particles are conceived as rigid spheres and their positions and velocities can take a high number of possible values. In the cellular automata-based model, the fluid particles are considered as points; the possible values of their positions and their velocities are forced to evolve within a discrete hexagonal lattice. Therefore, in a certain sense, the cellular automata-based model is an idealized version of the molecular dynamics model in which differences of mass are canceled and possible directions of velocity are limited.

The analysis of the different methods has shown that each method applies its own transformation with its own set of idealizations. Let me now illustrate that each transformation transforms the initially descriptive representation into a model that can be more or less adequate for the purpose at hand.

First, the macroscopic representations are not suitable for situations where Knudsen number is superior to 0,01 , but can be sufficiently accurate for other cases.

Second, unlike the discretized version of the Navier-Stokes equations, Prandtl's model is not sufficiently accurate for contemporary highly computerized studies in aeronautics or aerospace, but may be used to get an analytic understanding of how vortices appear at a rear of an obstacle.

Third, among microscopic representations, the Monte Carlo method enables one to make more rapid calculations, which can be sometimes required, but is not appropriate when it is about studying the onset of vortices. The statistics assumption that the Monte Carlo method involves has no major consequence when it is about modeling flows of Rayleigh-Stokes type (Meiburg 1986), but, as it fails to meet conservation of angular momentum and as angular momentum plays a central role for describing vortices, it may not be adapted for reproducing the onset of vortices at the rear of the obstacle. Here, molecular dynamics simulations are more adequate in order to reproduce the distributions of vortices (Meiburg 1986).

Fourth, cellular automata-based model is not adapted in cases where differences of mass within the fluid matter or where all directions of velocity must be taken into account. It may, however, be helpful in other cases.

Until now, I have argued that, for a model to be useful, the transformation has to be adapted to the nature of the target systems, and that, depending on the purpose at hand, it may be more adequate or less adequate. The question then arises: does this make the transformation idiosyncratic? I will offer an answer to this question in the final section.

\section{Scope of application}

Another usually expected property of tools is that they are to some extent generic. In other words, a tool is supposed to apply to some range of objects (rather than to a unique object). And yet if the transformation of models is idiosyncratic, i.e., if it 
is excessively adapted to the target system, it cannot be considered as the action of a tool. Note that the question is about the starting point, i.e., the systems to which it is applied, not the endpoint of the tool, i.e., into what it transforms. In this final section, I will argue that, even though a similar transformation does not strictly apply identically to all systems, the idealizations involved in the process may well be suited for a range of empirical systems. In that sense, idealizations may function as mathematical tools that have a certain scope of application.

I have suggested that idealizations give models inferential power in that they essentialize the features of the target(s) that they denote. They essentialize in the sense that they reduce the features to something formally and representationally essential. Therefore, an idealization that has been used in a model can be used in another model as soon as the two models share similar features to which the idealization is adapted.

This is the case with models used to study similar systems. In the previous example, the boundary layer applies to a class of hydrodynamics systems that share a certain physical feature, i.e., physical singularity. Examples are flow past a circle, flow over airfoil and flow over flat plate.

The boundary layer was initially used in a particular case as an idealization in order to make the Navier-Stokes equations analytically solvable. Today, the scope to which it adequately applies is much larger. It survived Prandtl's model and is now commonly used to describe concrete cases in fluid mechanics. It actually now belongs to the standard vocabulary of fluid mechanists and is a single-handedly research topic (see e.g., Khujadze et al. 2010).

The boundary layer has thus been mathematically defined as "a narrow region where the solution of a differential equation changes rapidly. By definition, the thickness of a boundary layer must approach 0 as [the perturbing parameter] $\varepsilon \rightarrow>$ 0" (Bender and Orszag 1978, p. 419). Therefore, as soon as a system has such a narrow region, the boundary layer may be an adequate idealization depending on the purpose at hand. This has also led to the development of the boundary layer theory which is "a collection of perturbation methods for solving differential equations whose solutions exhibit boundary-layer structure" (Bender and Orszag 1978, p. 420). The boundary layer is conceived more generally as leading to the following mathematical simplifications:

There are two standard approximations that one makes in boundary layer theory. In the outer region (away from a boundary layer) $\mathrm{y}(\mathrm{x})$ is slowly varying, so it is valid to neglect any derivatives of $\mathrm{y}(\mathrm{x})$ which are multiplied by $\varepsilon$. Inside a boundary layer the derivatives of $\mathrm{y}(\mathrm{x})$ are large, but the boundary layer is so narrow that we may approximate the coefficient functions of the differential equation by constants. Thus, we can replace a single differential equation by a sequence of much simpler approximate equations in each of several inner and outer regions. In every region the solution of the approximate equation will contain one or more unknown constants of integration. These constants are then determined from the boundary or initial conditions using the technique of asymptotic matching [...] (Bender and Orszag 1978, p. 421).

The boundary layer is here defined in a general way which includes the definition given by Prandtl in his model. This shows that the boundary layer is certainly 
not idiosyncratic but actually applies to a range of systems which share a common physical feature, i.e., discontinuity, that can be expressed mathematically in the previous terms.

On the same grounds, the scope of application of the boundary layer may actually extend beyond the range of hydrodynamics systems. It may also be a relevant idealization to describe the skin effect in electromagnetism, since this effect displays high variability. The skin effect is produced by an alternating electric current which has a high density within a conductor and is largest near the surface, while it decreases with greater depths in the conductor. The boundary layer can be associated with the skin depth in which the electric current flows ${ }^{10}$.

The scope of idealizations can more generally extend in case of formal analogy. In such a case, idealizations contained in a model A are transposed in a model B in that the equations in A are algebraically identical to the equations in B. This is a case of the analogies between waves of light, sound and water (Hesse 1966, p. 1012) where the same equation $y=a \sin (2 \pi f x)$ can be employed in the three cases ${ }^{11}$, between the atom and the solar system, between nuclear fission and the division of a liquid drop, or between electrostatic attraction and the conduction of heat (see Bailer-Jones 2009).

Therefore, idealizations function as tools in that they are adapted to typical empirical features of the investigated phenomena, which may be redundant in nature (e.g., oscillation, stochastic feature, discontinuity, etc.). They can thus be transposed to other cases which share a certain representational similarity. This should come as no surprise since, as said before, they reduce features of the phenomena to something formally and representationally essential. In their "toolbox," scientists may choose such or such a mathematical tool, ready to be used, depending on the system(s) under study.

In arguing that idealizations have a scope of application because they essentialize features of the target system, I do not want to suggest that they are parts of some mathematical structure of the empirical world or to support a platonic conception of idealizations. Rather, I want to claim that they can be used as tools because they have a story in the building of models and, as such, are recognized by scientists as being adequate for such or such modeling. A model is never built from scratch, but based on what is known to work in other models. For example, idealizing fluid as a continuum (rather than a discrete set of molecules) is a very common assumption in fluid mechanics models, as it is for other idealizations (e.g., incompressible flow assumption), since it has proven to be an adequate one.

\footnotetext{
10 The fact that idealizations have a certain scope of application may explain why some models are repeatedly used within and across scientific domains, e.g., the harmonic oscillator, the Ising model, a few Hamiltonians in quantum mechanics, the Poisson equation, or the Lokta-Volterra equations (see Barberousse and Imbert (2014) for an analysis of such recurring models).

${ }^{11}$ In the case of waves of water, the equation describes the height of the water at the point $\mathrm{x}$, with a being the maximum height or amplitude of the ripples, and $f$ their frequency. In the sound model, it describes the amplitude of a sound wave at the point $\mathrm{x}$, with a being the loudness and $\mathrm{f}$ the pitch. In the light model, it describes the amplitude of a light wave, with a being the brightness and $f$ the color.
} 
In other words, idealizations could be seen as tools in the scientific toolkit. Depending on the kind of target system, scientists could choose such or such idealization that is known to be adequate for modeling the system.

De-idealization may sometimes be required, however, in order to make the model sufficiently accurate (and therefore useful). It consists in adding features of the target (that were originally left out) back into the models and/or correcting idealizations that originally appear in the models (McMullin 1985; Laymon 1995). When de-idealization is required, it means that an additional aspect of the system is relevant for the purpose at hand that was not captured (or not properly captured) by the idealized model. This is the purpose at hand that determines which components are relevant to include in the model. Let me give an example.

In order to derive the ideal gas law $(P V=n R T)$ from a molecular model, gases are assumed to be perfectly elastic spherical molecules. These molecules exert no force and their volume is negligible in comparison with the volume occupied by the gas. These assumptions limit the application domain of the law which applies only to ranges of normal temperature and pressure. Thus, it is not possible to predict the properties of biphasic systems or monophasic systems that evolve towards a biphasic state (state transitions) with this law, which instead requires the use of van der Waals' equation. Van der Waals' equation, which is given by, $P+($ a/ $V 2)$ $(V-b)=R T$ (where $\mathrm{a}$ and $\mathrm{b}$ are associated with the intermolecular forces), is viewed as an improvement upon the ideal gas law because it takes into account attractive and repulsive intermolecular forces (see the chapter by Hasse and Lenhard which discusses the ideal gas law and the role of adjustable parameters in great detail). By adding the intermolecular forces, it yields more accurate results at high temperatures and low pressures than the ideal gas law, and therefore it applies to a wider domain than does the ideal gas law. The introduction of intermolecular forces into the new model is a genuine de-idealization (McMullin 1985) ${ }^{12}$.

To conclude, scientists may choose such or such idealization, depending on the system(s) under study and the purpose at hand. They can also decide to de-idealize in case the model is not sufficiently accurate.

\section{Conclusion}

I have first described how models are built, with special emphasis on the transformation. I have then argued that the transformation is always adapted to the target systems. The reason is that the idealizations involved in the process are both

\footnotetext{
${ }^{12}$ Some idealizations sometimes denote relevant aspects of the target model that de-idealization would fail to capture. These idealizations are called ineliminable (or essential) (Batterman 2005a, 2005b, 2009; Jones 2006; Sklar 2000). They cannot be removed without losing the explanation of the phenomenon that is studied. This is the case with the thermodynamic limit, according to which the number of atoms in the system is infinite, which is necessary for explaining phase transitions, and in particular, the phase transition of a magnet at a certain critical temperature (Batterman, 2005a).
} 
tool-related and object-related for the model to be useful. That said, I have further argued that adaptedness does not mean restriction to the sole systems under study. I have shown that the idealizations involved in a mathematical transformation may well be suited for other empirical systems as well and are in that sense mathematical tools that have a certain scope of application.

\section{References}

Bailer-Jones, D. M. (2009). Scientific models in philosophy of science. University of Pittsburgh Press.

Barberousse, A. and Imbert, C. (2014). Recurring Models and Sensitivity to Computational Constraints. The Monist, 97 (3), pp. 259-279.

Barberousse, A., Franceschelli, S. and Imbert, C. forthcoming. Cellular Automata, Modeling, and Computation.

Barberousse, A. and Imbert, C. (2013). New Mathematics for Old Physics: The Case of Lattice Fluids. Studies in History and Philosophy of Science Part B: Studies in History and Philosophy of Modern Physics, Elsevier, 44 (3), pp.231-241.

Batterman, B. (2010). On the Explanatory Role of Mathematics in Empirical Science. British Journal for the Philosophy of Science, 61:1-25.

Bender, C. M. \& Orszag, S. A. (1978). Advanced mathematical methods for scientists and engineers. McGraw-Hill Book Company.

Bokulich, A. (2008). Can Classical Structures Explain Quantum Phenomena? British Journal for the Philosophy of Science, 59(2):217-235.

Bokulich, A. (2009). Explanatory Fictions. In M. Suárez (Ed.) Fictions in Science: Philosophical Essays on Modeling and Idealization, (pp.91-109). Routledge, Londres.

Bueno, O. and Colyvan, M. (2011). An Inferential Conception of the Application of Mathematics. Noûs 45:2 345-374.

Cartwright, N. (1983). How the Laws of Physics Lie. Oxford University Press.

D'Alembert, J. (1782). Essai d'une nouvelle théorie de la résistance des fluides. Paris.

Darrigol, O. (2005). Worlds of flow: A history of hydrodynamics from the Bernoullis to Prandtl. Oxford: Oxford University Press.

D’Humières, D. \& Lallemand, P. (1986). Lattice gas automata for fluid me. Physica 140A 326335.

Godfrey-Smith, P. (2009). Models and Fictions in Science. Philosophical Studies, 143(1) 101116.

Hacking, I. (1983). Representing and Intervening: Introductory Topics in the Philosophy of Natural Science. Cambridge University Press.

Hesse, M. B. (1966). Models and Analogies in Science. University of Notre Dame Press.

Jones, M. R. (2005). Idealization and Abstraction: A Framework. In N. Cartwright, \& M. R. Jones (Eds.) Idealization XII: Correcting the model : idealization and abstraction in the sciences, (pp. 173-217). Amsterdam: Rodopi.

Keller, E. F. (2003). Models, simulation, and 'computer experiments. In H. Radder (Ed.) The philosophy of scientific experimentation, (pp. 198-215). Pittsburgh: University of Pittsburgh Press.

Khujadze, G., Nguyen van yen, R., Schneider, K., Oberlack, M., \& Farge, M. (2010). Coherent vorticity extraction in turbulent boundary layers using orthogonal wavelets. Bull. Amer. Phys. Soc., 55(16):206.

Laymon, R. (1980). Idealization, Explanation, and Confirmation. PSA: Proceedings of the Biennial Meeting of the Philosophy of Science Association, (pp. 336-350). 
Laymon, R. (1985). Idealizations and the Testing of Theories by Experimentation. In P. Achinstein, \& O. Hannaway (Eds.) Observation Experiment and Hypothesis in Modern Physical Science, (pp. 147-173). Cambridge, Mass.: M.I.T. Press.

Laymon, R. (1989a). Applying Idealized Scientific Theories to Engineering. Synthese, 81(3):353-371.

Laymon, R. (1989b). Cartwright and the Lying Laws of Physics. Journal of Philosophy, 86(7):353-372.

Laymon, R. (1995). Experimentation and the Legitimacy of Idealization. Philosophical Studies, 77(2-3):353-375.

McMullin, E. (1985). Galilean Idealization. Studies in History and Philosophy of Science Part A, 16(3):247-273.

Meiburg, E. (1986). Comparison of the molecular dynamics method and the direct simulation Monte Carlo technique for flows around simple geometries. Physics of Fluids, 29(10).

Morrison, M. (1999). Models as Autonomous Agents. In M. S. Morgan, \& M. Morrison (Eds.) Models as Mediators, (pp. 38-65). Cambridge, UK: Cambridge University Press.

Norton, J. D. (2012). Approximation and Idealization: Why the Difference Matters. Philosophy of Science, 79(2):207-232.

Pincock, C. (2007). Mathematical Idealization. Philosophy of Science, 74:957-967.

Ramsey, J. L. (1990). Beyond Numerical and Causal Accuracy: Expanding the Set of Justificational Criteria. Proceedings of the Biennial Meeting of the Philosophy of Science Association, 1:485-499.

Ramsey, J. L. (1992). Towards an Expanded Epistemology for Approximations. Proceedings of the Biennial Meeting of the Philosophy of Science Association, 1:154-164.

Rohrlich, F. (1991). Computer Simulation in the Physical Sciences. PSA 1990: Proceedings of the Biennial Meeting of the Philosophy of Science Association, 2:507-518.

Rothman, D., \& Zaleski, S. (2004). Lattice-Gas Cellular Automata. Simple models of complex hydrodynamics. Cambridge Univ. Press.

Strevens, M. (2007). Why explanations lie: Idealization in explanation. New York: New York University, unpublished manuscript.http://www.strevens.org/research/expln/Idealization.pdf

Van Dyke, M. (1975). Perturbation methods in fluid mechanics. The Parabolic Press, Stanford, California.

Weisberg, M. (2007). Three Kinds of Idealization. Journal of Philosophy, 104(12):639-659. 